\title{
Triggering Apoptotic Death of Human Epidermal Keratinocytes by Malic Acid: Involvement of Endoplasmic Reticulum Stress- and Mitochondria-Dependent Signaling Pathways
}

\author{
Yu-Ping Hsiao ${ }^{1,2}$, Wan-Wen Lai ${ }^{1}$, Shi-Bei Wu ${ }^{3}$, Chung-Hung Tsai ${ }^{1,4}$, Sheau-Chung Tang ${ }^{5,6}$, \\ Jing-Gung Chung ${ }^{7}$ and Jen-Hung Yang ${ }^{5,6, *}$
}

1 Institute of Medicine, Chung Shan Medical University, 402 Taichung, Taiwan;

E-Mails: missyuping@gmail.com (Y.-P.H.); hauhauma@yahoo.com.tw (W.-W.L.); pathology@csmu.edu.tw (C.-H.T.)

2 Department of Dermatology, Chung Shan Medical University Hospital, 402 Taichung, Taiwan

3 Department of Biochemistry and Molecular Biology, National Yang-Ming University, 112 Taipei, Taiwan; E-Mail: labboy700110@gmail.com

4 Department of Pathology, Chung Shan Medical University Hospital, 402 Taichung, Taiwan

5 Department of Dermatology, Buddhist Tzu Chi General Hospital, 907 Hualien, Taiwan;

E-Mail: s6160051@yahoo.com.tw

6 School of Medicine, Tzu Chi University, 907 Hualien, Taiwan

7 School of Biological Science and Biotechnology, China Medical University, 404 Taichung, Taiwan; E-Mail: jgchung@mail.cmu.edu.tw

* Author to whom correspondence should be addressed; E-Mail: jh.med.edu@ gmail.com; Tel.: +886-3-8565-301.

Academic Editor: Florian Lang

Received: 9 September 2014 / Accepted: 26 December 2014 / Published: 9 January 2015

\begin{abstract}
Malic acid (MA) has been commonly used in cosmetic products, but the safety reports in skin are sparse. To investigate the biological effects of MA in human skin keratinocytes, we investigated the potential cytotoxicity and apoptotic effects of MA in human keratinocyte cell lines (HaCaT). The data showed that MA induced apoptosis based on the observations of DAPI staining, DNA fragmentation, and sub-G1 phase in HaCaT cells and normal human epidermal keratinocytes (NHEKs). Flow cytometric assays also showed that MA increased the production of mitochondrial superoxide (mito-SOX) but decreased the mitochondrial membrane potential. Analysis of bioenergetics function with the XF 24 analyzer Seahorse extracellular flux analyzer demonstrated that oxygen consumption rate
\end{abstract}


(OCR) was significantly decreased whereas extracellular acidification rate (ECAR) was increased in MA-treated keratinocytes. The occurrence of apoptosis was proved by the increased expressions of FasL, Fas, Bax, Bid, caspases-3, -8, -9, cytochrome $c$, and the declined expressions of Bcl-2, PARP. MA also induced endoplasmic reticulum stress associated protein expression such as GRP78, GADD153, and ATF6 $\alpha$. We demonstrated that MA had anti-proliferative effect in HaCaT cell through the inhibition of cell cycle progression at G0/G1, and the induction of programmed cell death through endoplasmic reticulum stress- and mitochondria-dependent pathways.

Keywords: malic acid (MA); HaCaT cells; seahorse XF 24 analyzer; apoptosis

\section{Introduction}

Malic acid (MA), a sort of alpha-hydroxy acids (AHAs) found in fruits and many vegetables, has been commonly used in cosmetics and chemical peeling agents [1-4]. Malic acid is used for light-damaged or dry skin, and acne [3]. Over 50 cosmetic formulations across a range of products have contained MA [4]. However, safety concerns of the adverse reactions of AHAs including redness, swelling, burning, pruritus, phototoxicity, and facial hyperkeratosis were pronounced [2,5-8]. Malic acid may induce skin and ocular irritation [4] and the biological and molecular effects of malic acid in human keratinocytes are still uncertain. In this study, we investigated the potential cytotoxicity and apoptotic effects of malic acid in human keratinocyte cell lines (HaCaT) and normal human epidermal keratinocytes (NHEKs).

\section{Results}

\subsection{Anti-Proliferative Effects of MA on Cell Morphology and Viability of HaCaT Cells}

$\mathrm{HaCaT}$ cells were treated with MA at various concentrations $(0,10,12.5,15,17.5$, and $20 \mathrm{mM})$ for $24 \mathrm{~h}$ and $48 \mathrm{~h}$, and at $15 \mathrm{mM}$ for different incubation periods $(0,6,12,24$, and $48 \mathrm{~h})$. The cells were photographed and were collected before being stained by PI and were analyzed for viability by flow cytometry. A larger proportion of cells were swelling and round-out indicating necrosis, and some revealed shrinkage of cells with unclear nuclei suggesting apoptosis (Figure 1A). The effect of MA on viability of $\mathrm{HaCaT}$ cells revealed a time-dependent (Figure 1B) and dose-dependent manner (data not shown). The half maximal inhibitory concentration (IC50) value of MA was close to $15 \mathrm{mM}$ at a 24-h exposure. Thus, this concentration at $15 \mathrm{mM}$ of MA was applied for all subsequent experiments. 

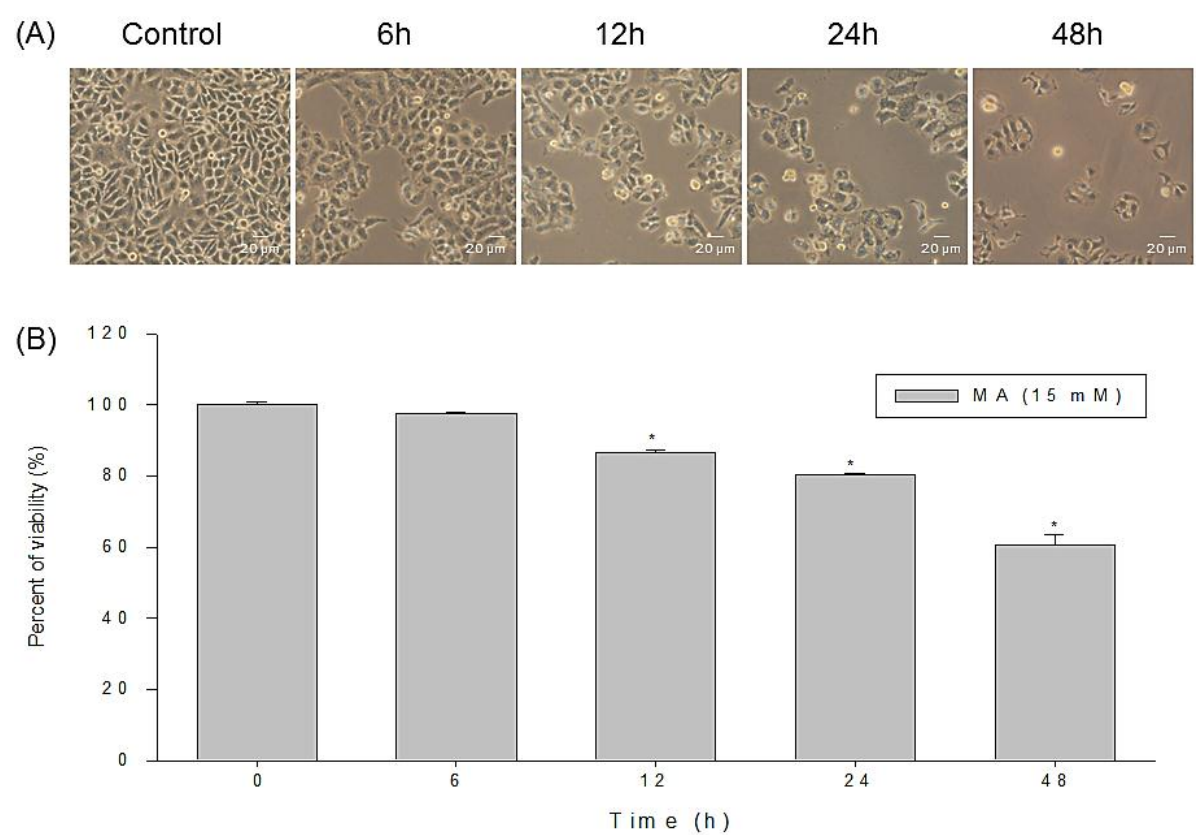

Figure 1. Malic acid induced cells' morphological changes and decreased the number of total viable human keratinocytes (HaCaT cells). Cells were incubated with or without $15 \mathrm{mM}$ of malic acid for $6,12,24$, and $48 \mathrm{~h}$, and then were examined and photographed by a phase-contrast microscope (A) and were harvested for determination the percentage of viable cells by flow cytometry $(\mathbf{B})$. Data are presented as means \pm S.D. of the results from three independent experiments $(* p<0.05 v s$. the indicated group).

\subsection{MA Induced Cell Cycle Arrest and Apoptosis in HaCaT Cells and NHEKs}

The flow cytometry disclosed that treatment of MA at $15 \mathrm{mM}$ increased the proportion of cells at G0/G1 phases after $24 \mathrm{~h}$ in HaCaT cells (Figure 2A). MA also induced a distinct subG1 peak, which represents the population of apoptotic cells in $\mathrm{HaCaT}$ cells and normal human epidermal keratinocytes (NHEKs) (Figure 2B).

\subsection{MA Induced Cell Damage Examined by DAPI Staining and DNA Fragmentation}

Apoptotic cells demonstrated with DAPI staining were higher in intensity than that in non-apoptotic live cells. There was an increase in the number of fragmented nuclei in MA-treated HaCaT cells at $24 \mathrm{~h}$ and $48 \mathrm{~h}$ (Figure 3A). MA-induced cell apoptosis was confirmed using a DNA fragmentation assay. HaCaT cells treated with $15 \mathrm{mM}$ of MA for 24 and $48 \mathrm{~h}$ showed a significant increase of DNA fragmentation compared to the controls (Figure 3B). 

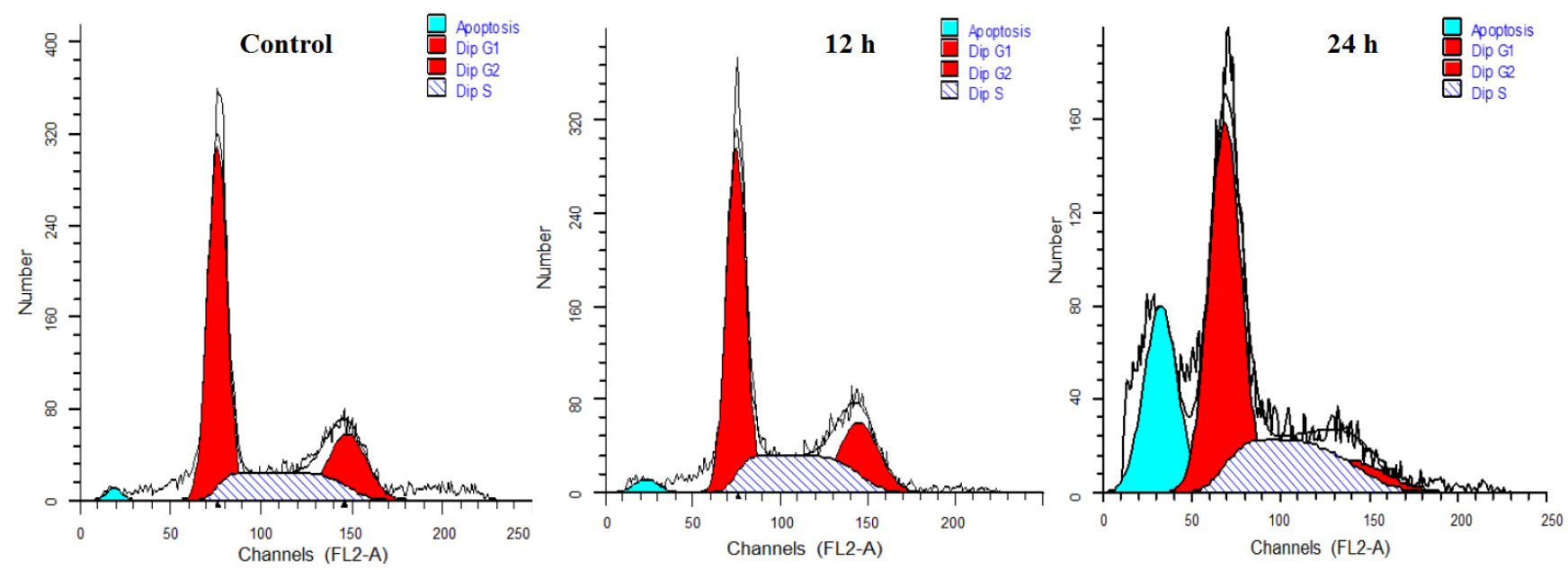

\begin{tabular}{llll} 
Cell cycle & Control & 12h & 24h \\
\hline G0/G1 phase & $56.09 \pm 0.66$ & $53.52 \pm 2.59$ & $62.63 \pm 1.12^{\star \star}$ \\
G2/M phase & $19.95 \pm 1.50$ & $20.78 \pm 0.34$ & $11.59 \pm 2.32$ \\
S phase & $23.97 \pm 0.83$ & $25.70 \pm 2.24$ & $25.78 \pm 3.44$ \\
Apoptosis & $1.42 \pm 0.17$ & $2.22 \pm 0.13$ & $27.26 \pm 4.24^{\star \star}$
\end{tabular}

(A)
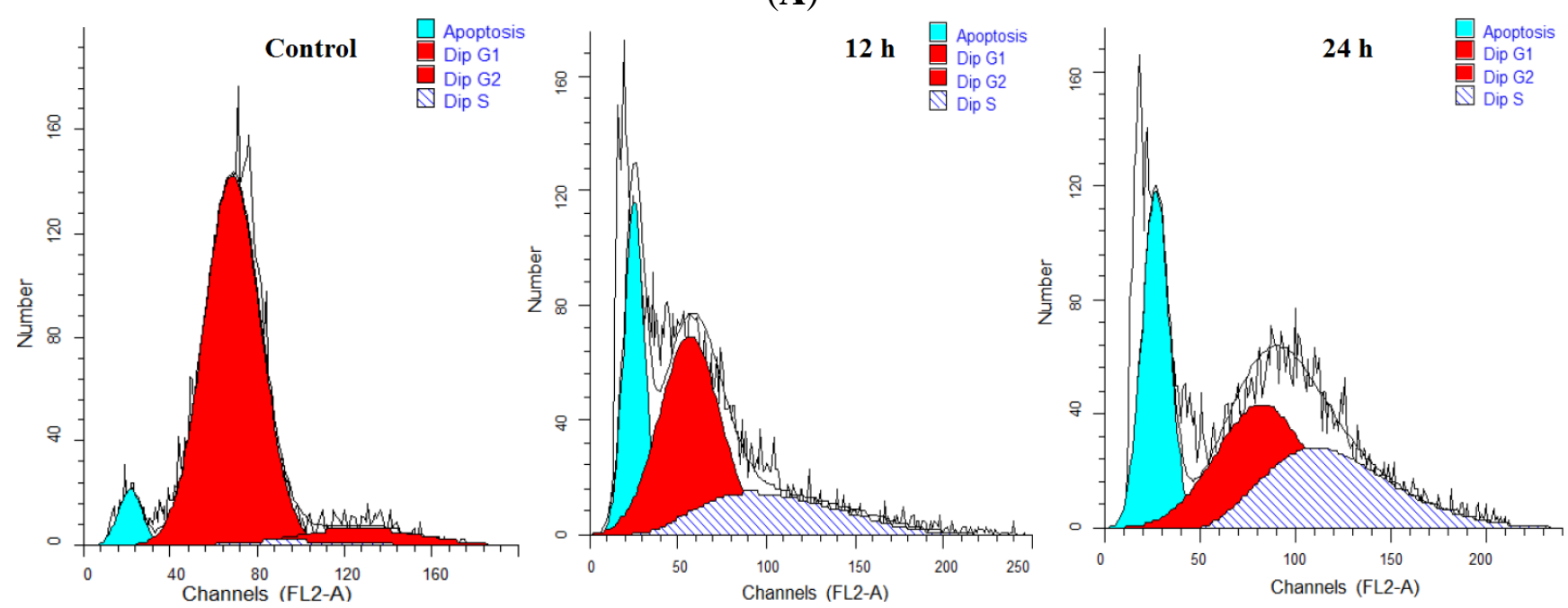

\begin{tabular}{l} 
Cell cycle \\
\hline G0/G1 phase \\
G2/M phase \\
S phase
\end{tabular}

12h

24h

Apoptosis

$88.51 \pm 1.66$

$62.85 \pm 2.80$

$54.31 \pm 1.96$

$9.30 \pm 1.14$

$4.75 \pm 3.28$

$2.37 \pm 0.97$

$S$ phase

$2.19 \pm 0.68$

$32.40 \pm 2.39$

$43.32 \pm 2.93$

$26.79 \pm 0.52^{\text {* }}$

$29.86 \pm 0.93^{\text {* }}$

(B)

Figure 2. Malic acid changed the DNA content and induced apoptosis in HaCaT cells (A); and normal human epidermal keratinocytes (NHEKs) (B). Cells were treated with $15 \mathrm{mM}$ of MA for 0,12 , and $24 \mathrm{~h}$. The cell cycle distribution and sub-G $\mathrm{G}_{1}$ group (apoptosis phase) were determined using flow cytometric analysis and obtained from three independent experiments with similar results. Data are presented as means \pm S.D. of the results from three independent experiments $(* * p<0.01 v s$. the indicated group). 
(A)

MA ( $15 \mathrm{mM})$

Control

$24 \mathrm{~h}$

$48 \mathrm{~h}$
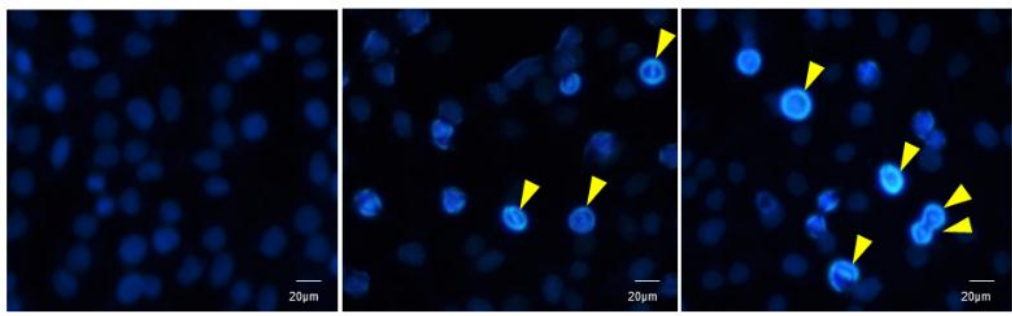

(B)

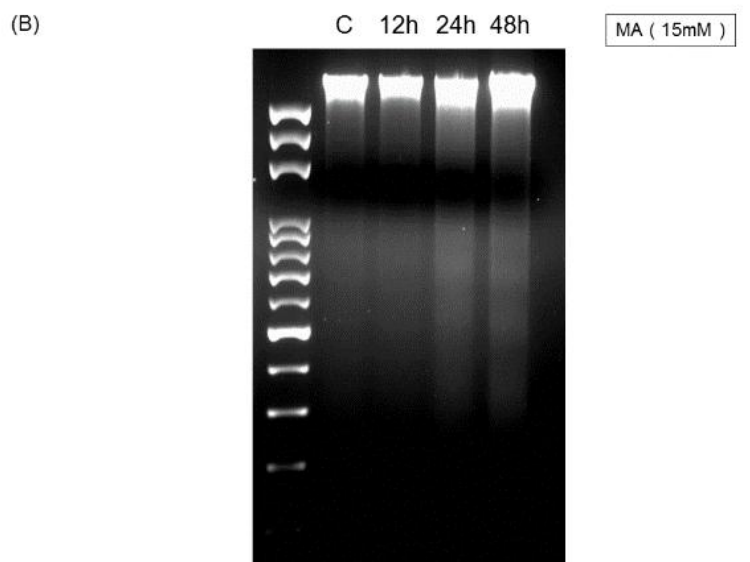

MA (15mM)

Figure 3. Effects of malic acid on DNA damage of HaCaT cells. Cells were treated with $15 \mathrm{mM}$ of malic acid for various time periods. Cells were harvested individually and then were measured with DAPI staining (A); and DNA gel electrophoresis (B) as described in Materials and Methods. Data are presented from three independent experiments.

\subsection{Intracellular Superoxide Dynamics of HaCaT Cells Incubated in Malic Acid}

$\mathrm{HaCaT}$ cells were incubated in malic acid dispersions and incubated with MitoSOX ${ }^{\mathrm{TM}}$ Red $-\mathrm{a}$ novel fluorescent indicator for the selective measurement of mitochondrial superoxide (O2--) production in cells (Invitrogen, Carlsbad, CA, USA). So, the effect of culturing HaCaT cells in malic acid (15 mM) medium for different time periods on mitochondrial superoxide (O2-•) was assessed using MitoSox. Under basal conditions, malic acid-cultured cells produced a significantly greater amount of mitochondrial superoxide $(\mathrm{O} 2 \cdot-)$ than those of control group in a time-dependent manner (Figure 4A). MA increased production of ROS in terms of increasing 2',7'-dichlorodihydrofluorescein (DCF) fluorescence intensity in HaCaT cells (Figure 4B).

\subsection{Measurement of Oxygen Consumption Rate (OCR) and Extracellular Acidification Rate (ECAR)} of Malic Acid-Treated HaCaT Cells by Seahorse XF24 Analyzer

To assess whether the malic acid had an effect on basal oxygen consumption and extracellular acidification in human skin, Seahorse XF24 Analyzer were used. There was no effect on basal oxygen consumption rate (OCR) and extracellular acidification rate (ECAR) of malic acid-treated HaCaT cells as compared to those of control without malic acid treatment. In addition, malic acid-treated HaCaT cells had higher mitochondrial stress in response to oligomycin and DNP $(p>0.05)$. Malic acid-treated HaCaT 
cells had lower OCR (more respiration) and greater glycolysis (ECAR) than control group $(p<0.05)$, suggesting greater glucose oxidation and glucose utilization in malic acid-treated HaCaT cells (Figure 4C).
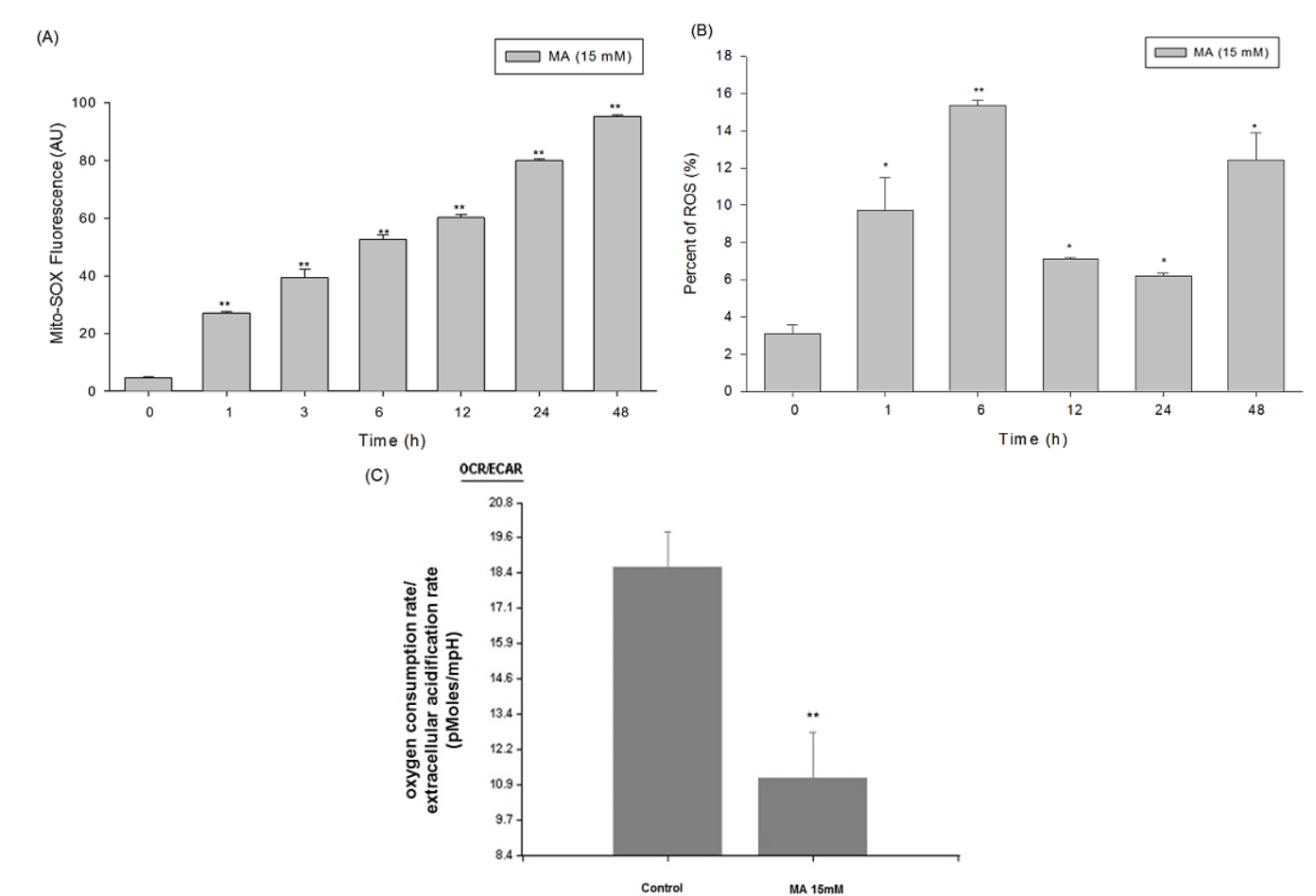

Figure 4. Increase of oxidative stress and anaerobic glycolysis in HaCaT cells treated with malic acid. (A) Malic acid increased mitochondrial ROS production in HaCaT cells detected by flow cytometry; (B) malic acid increased ROS production in terms of increasing 2',7'-dichlorodihydrofluorescein (DCF) fluorescence intensity in HaCaT cells detected by flow cytometry; (C) The mean values of oxygen consumption rate (OCR) and extracellular acidification rate (ECAR) were measured in real-time by a Seahorse XF24 Analyzer. Data are presented as means \pm S.D. of the results from three independent experiments $(* * p<0.01 v s$. the indicated group, *).

\subsection{Effects of MA on Caspase-3 and Inhibition of MA-Induced Apoptosis by the Caspase-3 Inhibitor Z-DEVD-FMK in HaCaT Cells}

MA affects the activities of caspase-8, -9 and -3 in HaCaT detected by cytometry (Figure S1). To confirm whether MA induced apoptosis through the activation of caspase- 3 in HaCat cells, cells were pretreated with or without the caspase-3 inhibitor (Z-DEVD-FMK) and then were treated with $15 \mathrm{mM}$ of MA and were harvested and assessed by flow cytometric assay and the results are shown in Figure 5A. These results showed that MA induced apoptosis via caspase-3 dependent pathway. Besides, This ROS was completely reduced in the presence of $N$-acetyl-L-cysteine (NAC) as shown in Figure 5B. 

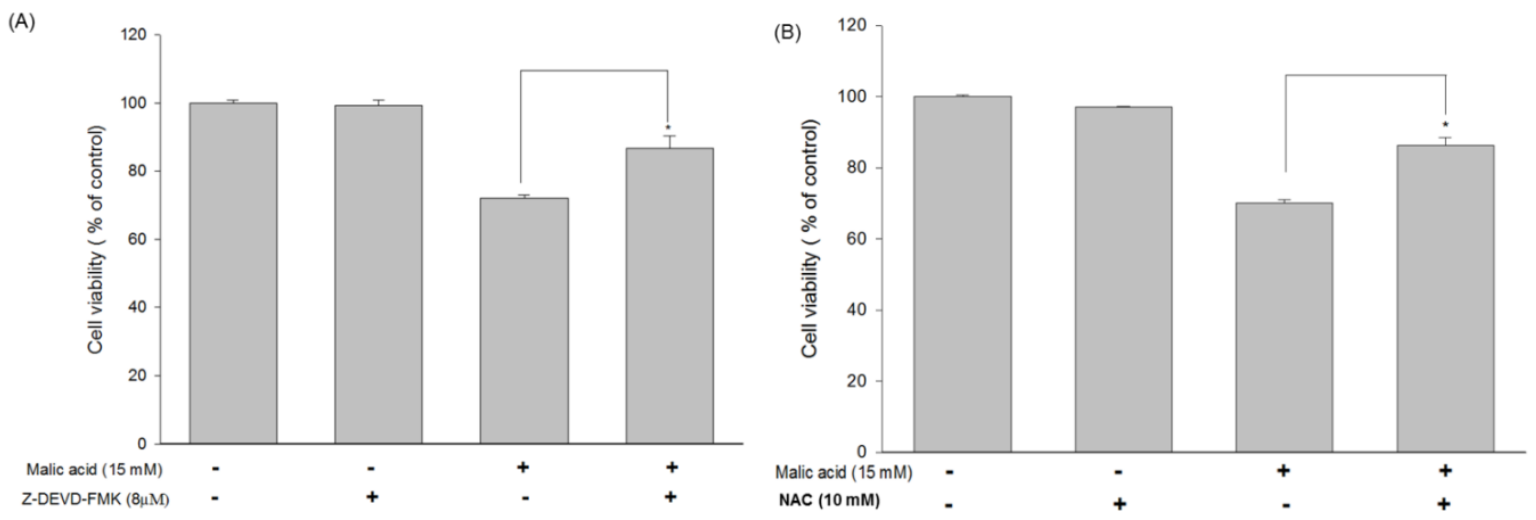

Figure 5. Malic acid stimulated ROS, caspase-3, -8 and -9 activities and altered viability after pre-incubation with specific inhibitors in HaCaT cells. (A) Cells were incubated with $15 \mathrm{mM}$ malic acid for $24 \mathrm{~h}$ before exposure in presence and absence of the specific inhibitors of caspase-3 (Z-DEVD-FMK) for $3 \mathrm{~h}$ to measure the viability in HaCaT cells; (B) Malic acid-treated $\mathrm{HaCaT}$ cells were in the presence and absence of $\mathrm{N}$-acetyl-L-cysteine (NAC) at a concentration of $10 \mathrm{mM}$. Cell viabilities were then determined as described above. Data are presented as means \pm S.D. of the results from three independent experiments (*p $0.05 v s$. the indicated group).

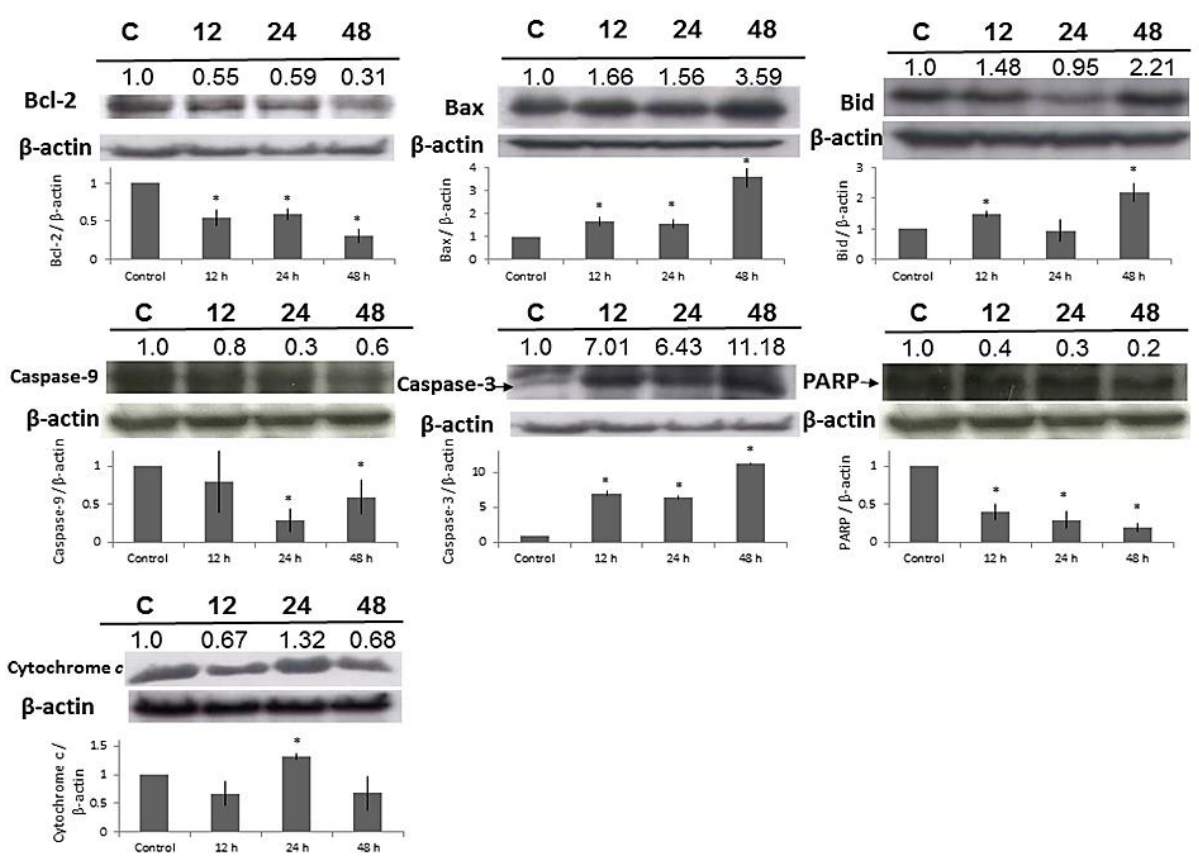

(A)

Figure 6. Cont. 

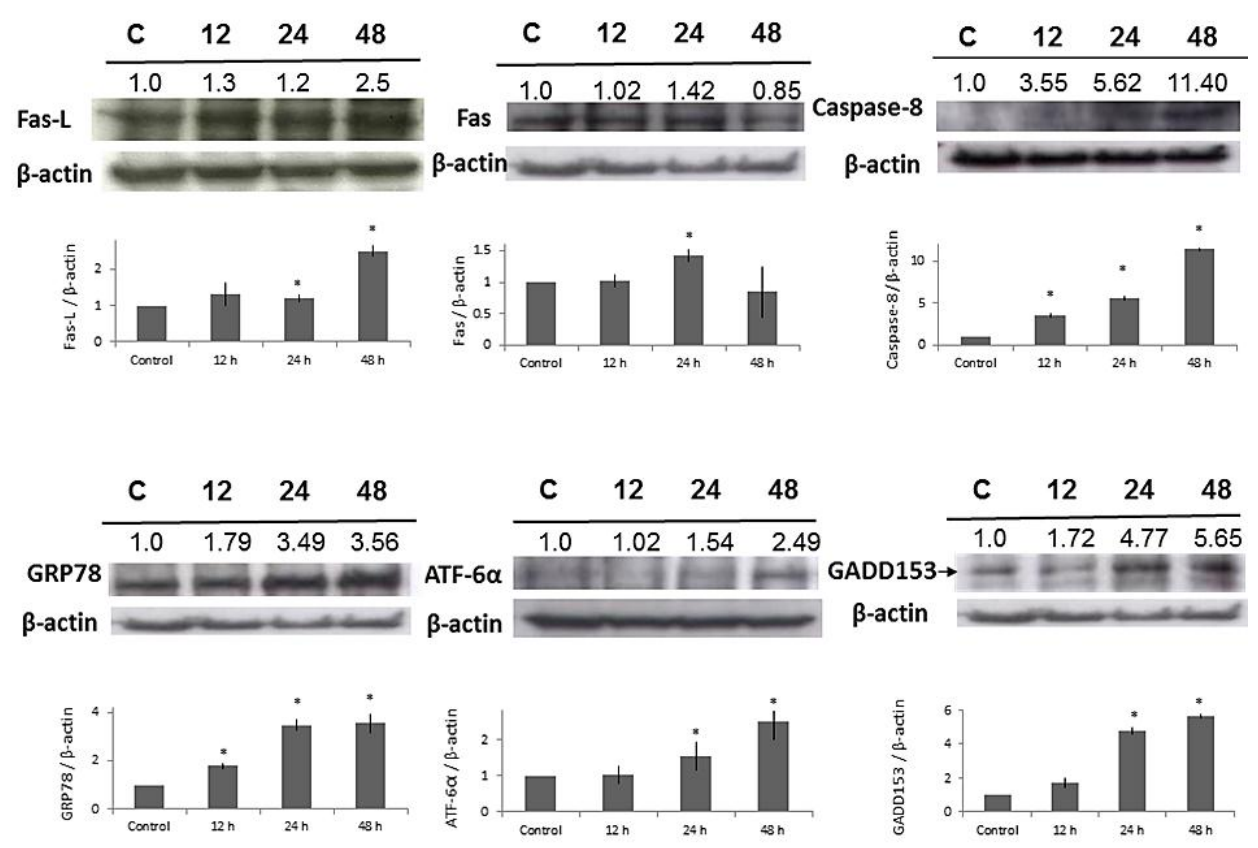

(B)
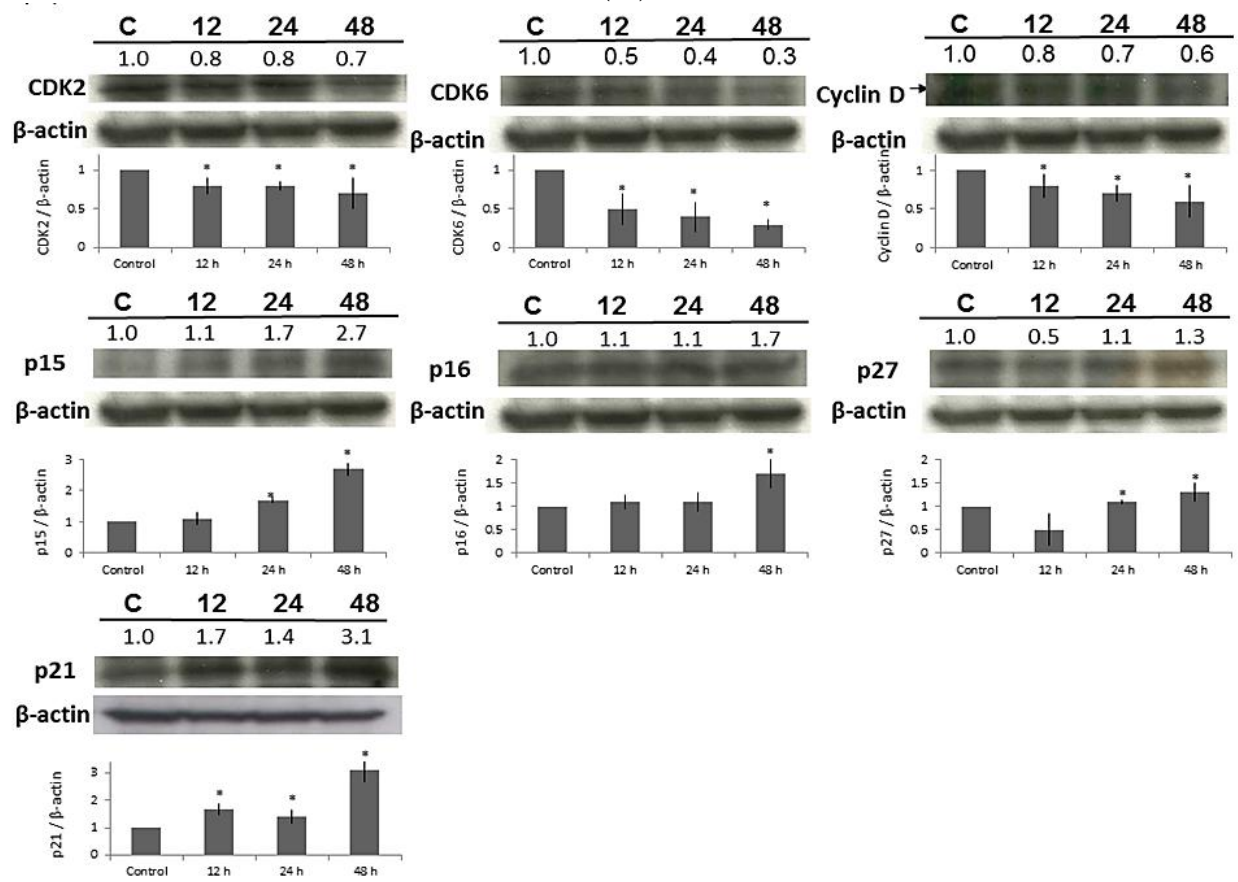

(C)

Figure 6. Western blot demonstrated the increase of Fas, FasL, Bax, Bid, caspase-3, -8, -9, cytochrome $c$, GADD153, GRP78, ATF6 $\alpha$, and decrease of Bcl-2 in HaCaT cells exposed to malic acid at $15 \mathrm{mM}$ for different incubation periods. Data are presented as means \pm S.D. of the results from three independent experiments $(* p<0.05 v s$. the control group).

\subsection{Effect of MA on the Expressions of Apoptosis-Related Proteins in HaCaT Cells}

$\mathrm{HaCaT}$ cells were treated with MA at $15 \mathrm{mM}$ for different time periods $(0,12,24$, and $48 \mathrm{~h})$. MA inhibited the levels of Bcl-2, PARP, but promoted the expressions of Bax, Bid, caspase-3, cytochrome c (Figure 6A). MA increased the expression of Fas-L, Fas, and caspase-8 and (Figure 6B). These findings 
show that MA induces apoptosis via the caspase- and mitochondrial-dependent pathway in HaCaT cells. Furthermore, MA promoted the expression of GRP78, ATF-6 $\alpha$, and GADD153 (Figure 6B), indicating that MA induced apoptosis through ER stress.

\subsection{Effect of MA on the Expressions of Cell Cycle-Related Proteins in HaCaT Cells}

Western blot demonstrated the decrease of CDK 2, CDK 6, and Cyclin D and the increase of P15, P16, P21, and P27; which indicated that MA induced cell cycle arrest at G0/G1 in MA-treated cells at $15 \mathrm{mM}$ for different periods $(0,12,24$, and $48 \mathrm{~h}$ ) (Figure 6C).

\section{Discussion}

Topical treatment of photo-aging skin with alpha-hydroxy acids (AHA) has been reported to improve wrinkling, roughness, hyperpigmentation, acne, and rosacea within months of daily application [9-12]. However, AHAs may induce photosensitivity, increase the number of sunburn cells, and decrease the minimal erythema dose [8,13-15]. Although the safety concerns of AHAs were announced by the Ministry of Health and Welfare, R.O.C, chemical peeling agents contained higher concentrations of AHAs (20\%-70\%) and low pH levels were used in the hospitals and local practitioner's clinics [16,17]. We have reported that glycolic acid induced programmed cell death in HaCaT cells and also human leukemia cell line HL-60 [8,18]. Although several reports have announced the adverse effects of AHAs, the exact molecular mechanisms of malic acid (MA) in human epidermal keratinocytes still remain unclear, and herein, we investigated MA affecting human epidermal keratinocytes (HaCaT cells).

In the present study, we firstly reported that MA exhibited cytotoxic effects towards HaCaT cells based on the observations of morphological changes (Figure 1A), and decreased the percentage of viable cells (Figure 1B), sub-G1 phase (apoptosis) (Figure 2) and DNA damage (Figure 3). Cells undergoing apoptosis and nuclear fragmentation were also observed in MA-treated cells and these effects are time-dependent.

Apoptosis (programmed cell death) belongs to the parts of DNA damage response [19]. The cellular reaction to genomic instability includes a network of signal transduction pathways referred to as the DNA damage response. Individual damage would trigger DNA damage response through distinct yet overlapping pathways to regulate the cell cycle, DNA repair, transcription, cell cycle progression, and apoptosis [19]. Apoptosis is regulated via the extrinsic pathway (death receptor-dependent pathway) and intrinsic pathway (mitochondria-dependent pathway) [20]. MA treatment promoted caspase-3 activity; however, co-administration of MA and caspase-3 inhibitor, Z-DEVD-FMK, markedly increased cell viability at $24 \mathrm{~h}$ in HaCaT cells (Figure 5A). The activation of caspase-3 is often considered as the point-of-no-return in the apoptotic signaling cascades [21].

The results from Western blotting (Figure 6A) indicated that MA increased endoplasmic reticulum stress associated protein expression such as GRP78, GADD153, and ATF6 $\alpha$ (Figure 6B). Based on the results of Western blotting (Figure 6A) and flow cytometry (Figure S1), MA also promoted the expression of Fas and FasL and active form of caspase-8; MA inhibited the protein level of Bcl-2 but promoted the Bax, and both proteins led to the release of cytochrome $c$ from mitochondria and activation of caspase-9 and -3, PARP cleavage, resulting in the apoptotic death which is mediated through the mitochondrial pathway. MitoSOX ${ }^{\mathrm{TM}}$ Red is a cell permeable dye that forms a highly fluorescent product 
upon oxidation [22,23]. Owing to its lipophilic triphenyl phosphonium cation, MitoSOX ${ }^{\mathrm{TM}}$ Red (Invitrogen, Carlsbad, CA, USA) is selectively targeted to mitochondria-the major source of ROS in cells - where it can be oxidized by superoxide before exhibiting red fluorescence upon binding to nucleic acids [22,23]. The XF 24 analyzer Seahorse is a novel extracellular flux analyzer used to analyze mitochondrial bioenergetics [24-26]. Mitochondrial oxygen consumption rate (OCR) is used to measure the oxidative phosphorylation and extracellular acidification rate (ECAR) as a measure of Glycolysis [24]. MA modulated mitochondrial biogenesis based on the fact that MA increased mitochondrial ROS MitoSOX ${ }^{\mathrm{TM}}$ Red (Invitrogen, Carlsbad, CA, USA) and decreased oxygen consumption rate/extracellular acidification rate in HaCaT (Figure 5).

In this study, the concentration of MA was $15 \mathrm{mM}(0.18 \%)$ and is remarkably lower than the concentration $(<1 \%)$ in cosmetics. Although the skin tissue levels of MA from the topical application of MA are lacking, our data clearly demonstrated that MA had anti-proliferative and apoptotic effects in human keratinocytes in vitro. Therefore, we are concerned about the potential adverse effects for long-term use of MA-containing resurfacing or peeling products. The limitation of our study is that $\mathrm{HaCaT}$ cells have intrinsic differences in comparison to those in primary normal human keratinocytes. The phases of cell cycle arrest were not similar in HaCaT cells and NHEKs (Figure 2B). Rate of cell proliferation in NHEKs was much slower than in HaCaT cells [27], and the percentage of G1-arrested cells was prominent in HaCaT cells than in NHEKs under oxidative stress [28]. That may explain part of reason why MA induced G0/G1 phases arrest in HaCaT cells, but caused S phase arrest in NHEKs. Nevertheless, NHEKs have a limited life span and could only survive for several passages. HaCaT is a convenient substitute and can maintain a non-tumorigenic phenotype [29]. HaCaT cells are excellent models to study the regulation of keratinocyte physiology by the circadian clock in vitro system [30].

In summary, we demonstrated that the effects of MA in HaCaT cells were as follows: (1) To cause cytotoxicity and morphological changes in immortalized human keratinocytes, and the inhibitory effect of MA in HaCaT viability was dose- and time-dependent; (2) To induce the cell cycle arrest at the G0/G1 transition via Cyclin D, CDK6, CDK2, P15, P16, P21, and P27 checkpoints; (3) To induce apoptosis based on the result of flow cytometry, DAPI staining, DNA gel electrophoresis, the induction of apoptosis was proved by the declined expressions of Bcl-2 and PARP and the increased expressions of ROS, Bax, Bid, caspases-3, -8, GRP78, GADD153, and ATF6a.

\section{Materials and Methods}

\subsection{Chemicals and Reagents}

Malic acid was obtained from Sigma Chemical (St. Louis, MO, USA). Dimethyl sulfoxide (DMSO), potassium phosphate, and TE buffer (10 mM Tris- $\mathrm{HCl}, \mathrm{pH} 8,1 \mathrm{mM}$ EDTA) were purchased from Merck Co. (Darmstadt, Germany). Trypan blue, Tris-HCl, triton X-100, propidium iodide (PI) and ribonuclease A were obtained from Sigma Chemical Co. (St. Louis, MO, USA). Fetal bovine serum, penicillin-streptomycin, trypsin-EDTA and glutamine were obtained from Gibco BRL (Grand Island, NY, USA). Caspase-3 activity assay kit was purchased from Roche Diagnostics (Mannheim, Germany). All of the chemicals used were reagent grade. 


\subsection{Human Immortalized Keratinocytes (HaCaT) Cell Line and Normal Human}

\section{Keratinocytes (NHEKs)}

HaCaT cells (Cell Lines Service, Eppelheim, Germany) were cultured on Dulbecco's modified Eagle's medium (DMEM) supplemented with 1\% L-glutamine, $25 \mathrm{mM}$ HEPES, $10 \%$ fetal bovine serum and $1 \%$ penicillin-streptomycin (Gibco, Carlsbad, CA, USA) at $37{ }^{\circ} \mathrm{C}$ in a humidified incubator with $5 \% \mathrm{CO}_{2}$ atmosphere [31]. Normal human epidermal keratinocytes (NHEKs) were obtained from Cell Applications, Inc. San Diego, USA and were cultured in Keratinocyte-SFM (Gibco BRL/Invitrogen, Carlsbad, CA, USA) supplemented with recombinant epidermal growth factor $(0.1-0.2 \mathrm{ng} / \mathrm{mL})$, bovine pituitary extract $(20-30 \mathrm{mg} / \mathrm{mL})$, and $1 \%$ penicillin/streptomycin in a humidified atmosphere at $37{ }^{\circ} \mathrm{C}$ and $5 \% \mathrm{CO} 2$. The second- to fourth-passage cells were used in the experiments.

\subsection{Morphological Changes and Viability of HaCaT Cells Treated with or without MA}

We observed the cell morphology under a phase-contrast microscope, and collected the HaCaT cells stained with PI and detected the viability with a flow cytometer (Becton-Dickinson, San Jose, CA, USA) equipped with an argon laser at $488 \mathrm{~nm}$ wavelength. We calculated the percentages of cell viability by using CellQuest software (Becton-Dickinson) and flow cytometer (Becton-Dickinson). Approximately $10 \mu \mathrm{L}$ of cell suspensions in PBS were mixed with $40 \mu \mathrm{L}$ of propidium iodine (PI), and the numbers of stained (dead cells) and unstained cells (live cells) were counted using flow cytometric assay. We used CellQuest to calculate the ratios of viable cells (PI negatively stained)/total cells as the percentages of cell viability. Data represent mean \pm SD of the results from three experiments $(n=3)$.

\subsection{Flow Cytometric Analysis of Cell Cycle and Apoptosis of HaCaT Cells and NHEKs Treated with Different Concentrations of MA}

HaCaT cells and normal human epidermal keratinocytes (NHEKs) were treated with MA (15 mM) in an incubator for different 0,12 , and $24 \mathrm{~h}$ before the cells were harvested by centrifugation. The cells were fixed gently by $70 \%$ ethanol at $4{ }^{\circ} \mathrm{C}$ for overnight and were then resuspended in PBS containing $40 \mathrm{mg} / \mathrm{mL}$ PI and $0.1 \mathrm{mg} / \mathrm{mL}$ RNase and $0.1 \%$ Triton X-100 in a dark room. After incubation at $37{ }^{\circ} \mathrm{C}$ for $30 \mathrm{~min}$, the cell cycles and apoptosis were analyzed with a flow cytometer.

\subsection{Examination of Apoptosis by 4,6-Diamidino-2-Phenylindole Dihydrochloride (DAPI) Staining and Determination of DNA Fragmentation by Gel Electrophoresis}

Approximately $2 \times 10^{5}$ cells/well of HaCaT cells had been treated with MA at $15 \mathrm{mM}$ for 0,24 , and $48 \mathrm{~h}$. Cells in each wells were stained with DAPI (4,6-diamidino-2-phenylindole dihydrochloride) before fixation with $3.7 \%$ formaldehyde. The cells were then washed with PBS and examined by fluorescence microscopy (Nikon Coolpix 4500, Tokyo, Japan, 200×).

Total DNA was isolated from each sample using a DNA purification kit (Genemark Technology, Tainan, Taiwan) and resolved in an $1.8 \%$ agarose gel containing $0.3 \mathrm{mg} / \mathrm{mL}$ ethidium bromide (Sigma-Aldrich, St. Louis, MO, USA) in a $0.5 \mathrm{X}$ TBE buffer $(0.045 \mathrm{~mol} / \mathrm{L}$ Tris, $0.045 \mathrm{~mol} / \mathrm{L}$ boric acid, $1 \mathrm{mmol} / \mathrm{L} \mathrm{Na} 2$ EDTA acid, $\mathrm{pH}$ 8.3) after electrophoresis for $45 \mathrm{~min}$. The DNA bands were visualized, examined, and photographed as described previously. 


\subsection{Determination of Mitochondrial ROS Production and Intracellular ROS by Flow Cytometry}

Cells were rinsed at least three times with sterile PBS and loaded with a solution of MitoSOX ${ }^{\mathrm{TM}}$ Red (Molecular Probes, Invitrogen, Carlsbad, CA, USA). Specifically, cells were incubated for $10 \mathrm{~min}$ at $37^{\circ} \mathrm{C}$ in a $5 \mu \mathrm{M}$ MitoSOX ${ }^{\mathrm{TM}}$ Red (Invitrogen, Carlsbad, CA, USA) solution. Next, cells were rinsed three times with PBS, harvested with $500 \mu \mathrm{L}$ of trypsin-EDTA solution, centrifuged at $5000 \mathrm{rpm}$ for $5 \mathrm{~min}$, and resuspended in $3 \mathrm{~mL}$ of fresh $2 \%(v / v)$ FBS/PBS. Finally, cell suspensions were filtered through a 30- $\mu \mathrm{m}$ PreSeparation filter (Miltenyi Biotec, Bergisch Gladbach, Germany). Fluorescence-based flow cytometry was performed using a Becton Dickinson FACSCalibur ${ }^{\circledR}$ (BD Biosciences, Totowa, NJ, USA) flow cytometer equipped with a $488 \mathrm{~nm}$ laser. MitoSOX ${ }^{\mathrm{TM}}$ Red (Invitrogen, Carlsbad, CA, USA) fluorescence $(\lambda \operatorname{Max}=590 \mathrm{~nm})$ was detected over the range of 564-606 nm and the background fluorescence was detected over the range of 515-545 nm. Besides, the levels of intracellular ROS of the $\mathrm{HaCaT}$ cells were determined by flow cytometry. HaCaT cells were treated with or without MA (15 mM) for different time periods $(0,1,6,12,24$, and $48 \mathrm{~h})$. The cells were harvested and washed twice, resuspended in $10 \mu \mathrm{M}$ 2,7-dichlorodihydrofluorescein diacetate (Sigma-Aldrich, St. Louis, MO, USA) and incubated at $37^{\circ} \mathrm{C}$ for $30 \mathrm{~min}$ and the levels of ROS were analyzed by flow cytometry. Intracellular ROS was detected by means of an oxidation-sensitive fluorescent probe (DCFH-DA). In the presence of ROS, DCFH-DA was subsequently transferred to DCF and emitted a green fluorescent signal detected by flow cytometry. All quantitations were performed using CellQuest 7.5.3 software (BD Biosciences, Totowa, NJ, USA); in each experiment, well over 10,000 cells were analyzed.

\subsection{Measurement of Bioenergetic Parameters of Mitochondria by Seahorse XF24 Analyzer}

An XF24 Analyzer (Seahorse Bioscience, North Billerica, MA, USA) was used to measure the bioenergetic function of the primary culture of skin fibroblasts [25]. The XF24 Analyzer can create a transient $7-\mu \mathrm{L}$ chamber to the cells cultured in a 24 -well microplate, and the oxygen consumption rate (OCR) and extracellular acidification rate (ECAR) were monitored real-time in an incubation chamber at $37^{\circ} \mathrm{C}$. Briefly, a seeding density of 16,000 skin cells per well was chosen and the culture medium was replaced $1 \mathrm{~h}$ prior to measurement by the assay medium that contained un-buffered DMEM (pH 7.4). The program of Seahorse XF24 Analyzer was set according to the manufacturer's recommendation and the data are expressed in $\mathrm{pmol} / \mathrm{min} / 10^{4}$ cells for OCR and in $\mathrm{mpH} / \mathrm{min} / 10^{4}$ cells for ECAR, respectively, to allow comparison between independent experiments.

\subsection{Determination the Effect of Caspase-3 Inhibitor Z-DEVD-FMK and ROS Inhibitor NAC on Cell Viability in MA-Treated HaCaT Cells}

HaCaT cells were treated with MA $(15 \mathrm{mM})$ for $24 \mathrm{~h}$, and then with lysis buffer (1\% Triton X-100, $0.32 \mathrm{M}$ sucrose, $5 \mathrm{mM}$ EDTA, $10 \mathrm{mM}$ Tris-HCl, $\mathrm{pH}$ 8, $2 \mathrm{mM}$ dithiothreitol, $1 \mathrm{mM}$ phenylmethylsulfonyl fluoride, $1 \mu \mathrm{g} / \mathrm{mL}$ aprotinin, and $1 \mathrm{mg} / \mathrm{mL}$ leupeptin) for $30 \mathrm{~min}$ at $4{ }^{\circ} \mathrm{C}$ followed by centrifugation at $10,000 \times g$ for $30 \mathrm{~min}$. To examine whether or not caspase-3 activation was involved in cell viability triggered by MA, HaCaT cells had been pretreated with Z-DEVD-FMK (caspase-3 inhibitor) $3 \mathrm{~h}$ prior to treatment with MA. MA-treated $\mathrm{HaCaT}$ cells were in the presence and absence of $N$-acetyl-L-cysteine (NAC) at a concentration of $10 \mathrm{mM}$. Cell viabilities were then determined as described above [7]. 


\subsection{Western Blotting for Examining the effect of MA on Expressions of Apoptosis-Related and Cell Cycle Checkpoints-Related Proteins}

Total proteins were collected from HaCaT cells treated with MA for different time period $(0,12,24$, and $48 \mathrm{~h}$ ). Western blotting was used to examine the expression levels of the apoptosis-related proteins including Bax, Bcl-2, Bid, PARP, caspases-3, caspase-4, caspases-8, caspase-9, cytochrome $c$, Fas, Fas-L, ATF-6 $\alpha$, GADD153, GRP 78, and cell cycle-related proteins including CDK 2, CDK 6, cyclin D, P15, P16, P21, P27 by sodium dodecylsulfate polyacrylamide gel electrophoresis (SDS-PAGE). The protein bands were visualized and measured by using Alpha Image 1220 Documentation and Analysis System (Alpha Innotech, San Leandro, CA, USA).

\subsection{Statistical Analysis}

All experiments were performed in triplicate and presented as means \pm SD. Statistical analyses were performed using one-way analysis of variance (ANOVA, SAS software, SAS Institute Inc., Cary, NC, USA, version 9.2). followed by Tukey post hoc test to determine significant differences among the groups. The difference was considered significant when $p<0.05$.

\section{Conclusions}

In conclusion, we demonstrated that MA induced cytotoxic effects and anti-proliferative effect in $\mathrm{HaCaT}$ cell through the inhibition of cell cycle progression and the induction of programmed cell death in vitro. We also demonstrated that MA-induced apoptosis were through multiple molecular pathways including involvement of endoplasmic reticulum stress- and mitochondria-dependent signaling pathways (Figure 7).

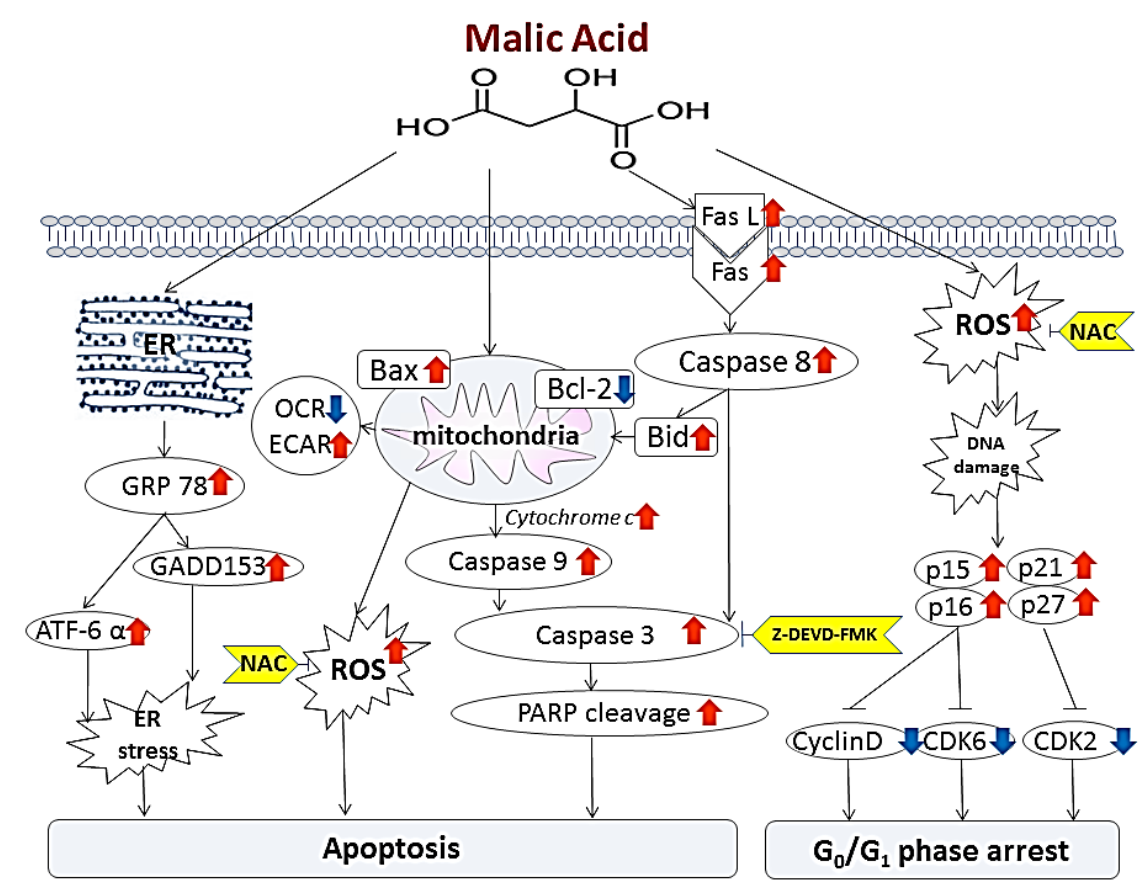

Figure 7. The proposed signaling pathways of malic acid-induced G0/G1 phase arrest and apoptosis in human epidermal keratinocytes HaCaT cells. 


\section{Supplementary Materials}

Supplementary materials can be accessed at: http://www.mdpi.com/2072-6651/7/1/0081/s1.

\section{Acknowledgments}

This work was supported by the grant NSC97-2314-B-040-026, NSC102-2314-B-303-002 from the National Science Council, Taiwan, Republic of China, the grant TCRD102-42, TCRD103-03 from Buddhist Tzu Chi General Hospital, and the grant CSH-2012-C-014 from Chung Shan Medical University Hospital, Taiwan.

\section{Author Contributions}

Conceived and designed the experiments: Y.-P.H., J.-H.Y.; Performed the experiments: Y.-P.H., W.-W.L., S.-B.W.; Analyzed the data: Y.-P.H., C.-H.T., S.-C.T., J.-G.C. and J.-H.Y.; Contributed reagents/materials/analysis tools: Y.-P.H., W.-W.L., C.-H.T. and J.-G.C.; Wrote the paper: Y.-P.H., J.-H.Y.

\section{Conflicts of Interest}

The authors declare no conflict of interest.

\section{References}

1. Taylor, M.B.; Yanaki, J.S.; Draper, D.O.; Shurtz, J.C.; Coglianese, M. Successful short-term and long-term treatment of melasma and postinflammatory hyperpigmentation using vitamin $\mathrm{C}$ with a full-face iontophoresis mask and a mandelic/malic acid skin care regimen. J. Drugs Dermatol. 2013, $12,45-50$.

2. Ramos-e-Silva, M.; Celem, L.R.; Ramos-e-Silva, S.; Fucci-da-Costa, A.P. Anti-aging cosmetics: Facts and controversies. Clin. Dermatol. 2013, 31, 750-758.

3. Al-Bawab, A.; Friberg, S.E.; Bergamaschi, M.M.; Santos, O.D. Some non-equilibrium phenomena in the malic acid/water/Polysorbate 81 system. Int. J. Pharm. 2007, 332, 140-146.

4. Fiume, Z. Final report on the safety assessment of Malic Acid and Sodium Malate. Int. J. Toxicol. 2001, 20, 47-55.

5. Kurtzweil, P. Alpha hydroxyl acids for skin care: Smooth sailing or rough seas? FDA Consumer. 1998, 32, 30-35.

6. Chou, T.C.; Chang, C.H. A case of facial hyperkeratosis induced by a cosmeceutical containing alpha-hydroxy acid and sunlight overexposure successfully treated using oral acitretin. Dermatol. Sinica 2013, 31, 161-162.

7. Hsiao, Y.P.; Huang, H.L.; Lai, W.W.; Chung, J.G.; Yang, J.H. Antiproliferative effects of lactic acid via the induction of apoptosis and cell cycle arrest in a human keratinocyte cell line (HaCaT). J. Dermatol. Sci. 2009, 54, 175-184.

8. Lai, W.W.; Hsiao, Y.P.; Chung, J.G.; Wei, Y.H.; Cheng, Y.W.; Yang, J.H. Synergistic phototoxic effects of glycolic acid in a human keratinocyte cell line (HaCaT). J. Dermatol. Sci. 2011, 64, 191-198. 
9. Green, B.A.; Yu, R.J.; van Scott, E.J. Clinical and cosmeceutical uses of hydroxyacids. Clin. Dermatol. 2009, 27, 495-501.

10. Bilaç C.; Şahin, M.T.; Öztürkcan, S. Chronic actinic damage of facial skin. Clin. Dermatol. 2014, in press.

11. Usuki, A.; Ohashi, A.; Sato, H.; Ochiai, Y.; Ichihashi, M.; Funasaka, Y. The inhibitory effect of glycolic acid and lactic acid on melanin synthesis in melanoma cells. Exp. Dermatol. 2003, 12, $43-50$.

12. Tung, R.C.; Bergfeld, W.F.; Vidimos, A.T.; Remzi, B.K. $\alpha$-Hydroxy Acid-Based Cosmetic Procedures. Guidelines for patient management. Am. J. Clin. Dermatol. 2000, 1, 81-88.

13. Kaidbey, K.; Sutherland, B.; Bennett, P.; Wamer, W.G.; Barton, C.; Dennis, D.; Kornhauser, A. Topical glycolic acid enhances photodamage by ultraviolet light. Photodermatol. Photoimmunol. Photomed. 2003, 19, 21-27.

14. Hong, J.T.; Kim, E.J.; Ahn, K.S.; Jung, K.M.; Yun, Y.P.; Park, Y.K.; Lee, S.H. Inhibitory effect of glycolic acid on ultraviolet-induced skin tumorigenesis in SKH-1 hairless mice and its mechanism of action. Mol. Carcinog. 2001, 31, 152-160.

15. Ahn, K.S.; Park, K.S.; Jung, K.M.; Jung, H.K.; Lee, S.H.; Chung, S.Y.; Yang, K.H.; Yun, Y.P.; Pyo, H.B.; Park, Y.K.; et al. Inhibitory effect of glycolic acid on ultraviolet B-induced c-fos expression, AP-1 activation and p53-p21 response in a human keratinocyte cell line. Cancer Lett. 2002, 186, 125-135.

16. Ying, T.H.; Chen, C.W.; Hsiao, Y.P.; Hung, S.J.; Chung, J.G.; Yang, J.H. Citric acid induces cell-cycle arrest and apoptosis of human immortalized keratinocyte cell line (HaCaT) via caspaseand mitochondrial-dependent signaling pathways. Anticancer Res. 2013, 33, 4411-4420.

17. Taiwan Ministry of Health and Welfare Commercially Available Alpha-Hydroxy Acids-Containing Cosmetic Ingredients Have complete Management Practices. Available online: http://cosmetic-recall.fda.gov.tw (accessed on 9 September 2014).

18. Yang, J.H.; Chou, C.C.; Cheng, Y.W.; Sheen, L.Y.; Chou, M.C.; Yu, H.S.; Wei, Y.H.; Chung, J.G. Effects of glycolic acid on the induction of apoptosis via caspase-3 activation in human leukemia cell line (HL-60). Food Chem. Toxicol. 2004, 42, 1777-1784.

19. Hsiao, Y.P.; Yu, C.S.; Yu, C.C.; Yang, J.S.; Chiang, J.H.; Lu, C.C.; Huang, H.Y.; Tang, N.Y.; Yang, J.H.; Huang, A.C.; et al. Triggering apoptotic death of human malignant melanoma a375.s2 cells by bufalin: involvement of caspase cascade-dependent and independent mitochondrial signaling pathways. Evid. Based Complement. Altern. Med. 2012, doi:10.1155/2012/591241.

20. Raleigh, D.R.; Haas-Kogan, D.A. Molecular targets and mechanisms of radiosensitization using DNA damage response pathways. Future Oncol. 2013, 9, 219-233.

21. Green, D.R.; Amarante-Mendes, G.P. The point-of-no-return: Mitochondria, caspases, and the commitment to cell death. Results Probl. Cell Differ. 1998, 24, 45-61.

22. Julian, D.; April, K.L.; Patel, S.; Stein, J.R.; Wohlgemuth, S.E. Mitochondrial depolarization following hydrogen sulfide exposure in erythrocytes from a sulfide-tolerant marine invertebrate. J. Exp. Biol. 2005, 208, 4109-4122.

23. Yehia, H.N.; Draper, R.K.; Mikoryak, C.; Walker, E.K.; Bajaj, P.; Musselman, I.H.; Daigrepont, M.C.; Dieckmann, G.R.; Pantano, P. Single-walled carbon nanotube interactions with HeLa cells. J. Nanobiotechnol. 2007, 5, 8. 
24. Ferrick, D.A.; Neilson, A.; Beeson, C. Advances in measuring cellular bioenergetics using extracellular flux. Drug Discov. Today 2008, 13, 268-274.

25. Wu, S.B.; Wei, Y.H. AMPK-mediated increase of glycolysis as an adaptive response to oxidative stress in human cells: Implication of the cell survival in mitochondrial diseases. Biochim. Biophys. Acta 2012, 1822, 233-247.

26. Nicholls, D.G.; Darley-Usmar, V.M.; Wu, M.; Jensen, P.B.; Rogers, G.W.; Ferrick, D.A. Bioenergetic profile experiment using C2C12 myoblast cells. J. Vis. Exp. 2010, 46, 2511.

27. Hail, N., Jr.; Chen, P.; Kepa, J.J.; Bushman, L.R. Evidence supporting a role for dihydroorotate dehydrogenase, bioenergetics, and p53 in selective teriflunomide-induced apoptosis in transformed versus normal human keratinocytes. Apoptosis 2012, 17, 258-268.

28. Liu, L.; Xie, H.; Chen, X.; Shi, W.; Xiao, X.; Lei, D.; Li, J. Differential response of normal human epidermal keratinocytes and $\mathrm{HaCaT}$ cells to hydrogen peroxide-induced oxidative stress. Clin. Exp. Dermatol. 2012, 37, 772-780.

29. Fusenig, N.E.; Boukamp, P. Multiple stages and genetic alterations in immortalization, malignant transformation, and tumor progression of human skin keratinocytes. Mol. Carcinog. 1998, 23, 144-158.

30. Spörl, F.; Schellenberg, K.; Blatt, T.; Wenck, H.; Wittern, K.P.; Schrader, A.; Kramer, A. A circadian clock in HaCaT keratinocytes. J. Investig. Dermatol. 2011, 131, 338-348.

31. Boukamp, P.; Petrussevska, R.T.; Breitkreutz, D.; Hornung, J.; Markham, A.; Fusenig, N.E. Normal keratinization in a spontaneously immortalized aneuploid human keratinocyte cell line. J. Cell Biol. 1988, 106, 761-771.

(C) 2015 by the authors; licensee MDPI, Basel, Switzerland. This article is an open access article distributed under the terms and conditions of the Creative Commons Attribution license (http://creativecommons.org/licenses/by/4.0/). 